\title{
Identification of Urdu Ghazal Poets using SVM
}

\author{
NIDA TARIQ*, IQRA EJAZ*, MUHAMMAD KAMRAN MALIK*, ZUBAIR NAWAZ*, AND \\ FAISAL BUKHARI*
}

RECEIVED ON 08.06.2018 ACCEPTED ON 30.10.2018

\begin{abstract}
Urdu literature has a rich tradition of poetry, with many forms, one of which is Ghazal. Urdu poetry structures are mainly of Arabic origin. It has complex and different sentence structure compared to our daily language which makes it hard to classify. Our research is focused on the identification of poets if given with ghazals as input. Previously, no one has done this type of work. Two main factors which help categorize and classify a given text are the contents and writing style. Urdu poets like Mirza Ghalib, Mir Taqi Mir, Iqbal and many others have a different writing style and the topic of interest. Our model caters these two factors, classify ghazals using different classification models such as SVM (Support Vector Machines), Decision Tree, Random forest, Naïve Bayes and KNN (K-Nearest Neighbors). Furthermore, we have also applied feature selection techniques like chi square model and L1 based feature selection. For experimentation, we have prepared a dataset of about 4000 Ghazals. We have also compared the accuracy of different classifiers and concluded the best results for the collected dataset of Ghazals.
\end{abstract}

Key Words: Text classification, Support Vector Machines, Urdu poetry, Naïve Bayes, Decision Tree, Feature Selection, Chi Square, k-Nearest Neighbors, Ghazal, L1, Random Forest.

\section{INTRODUCTION}

$\mathrm{P}$ oetry is a form of literature in which a poet expresses his feelings in a rhythmic manner. Great to the literature on different themes with their unique writing style. Since the $17^{\text {th }}$ century, when Urdu was declared the official language, we have seen many famous poets as the likes of Ghalib, Meer, Anees, Dard, Daag, Zauq, Iqbal, Akbar, Josh, Firaq, Faiz and Faraz to name a few [1]. Urdu has a rich tradition of poetry and has many forms. Ghazal is one of the most famous forms of poetry in Urdu, which describes love in a rhythmic way [2]. Like poetry in other languages, love has been the most popular topic amongst Urdu poets. An interesting work is to identify poets from the given poetry, since each poet has a different style of writing and content. Poet identification may help us to fight plagiarism.

A very little work has been done in poetry classification in any language. In the Malay language, Noraini et. al. [3] have classified the theme of the poetry. The apparent reason for little work done in Urdu and Urdu poetry is its different and complex language structure. As poetry can

Authors E-Mail: (mscsf16m005@pucit.edu.pk, iqraijaz44@gmail.com,kamran.malik@pucit.edu.pk, z.nawaz@pucit.edu.pk, faisal.bukhari@pucit.edu.pk)

* College of Information Technology, University of the Punjab, Lahore, Pakistan.

This is an open access article published by Mehran University Research Journal of Engineering and Technology, Jamshoro under the CC by 4.0 International License. 
be very different in terms of lines, stanzas, rhyme and style of rhythm. Classifying Urdu poetry thus is much trickier.

This paper presents the evaluation experiments on the performance of various machine learning algorithms as the likes of SVM [4-5], KNN [6], Random forest [7], Decision Tree [7] and Naïve Bayes [8-9] for Urdu poet identification. The application of machine learning is now very common in classification and identification of text, e.g. classifying emails as spam [10], identifying authors in English text [11] and text classification of news in Urdu [12]. In this study, we have focused on poet identification in Ghazals only. Ghazals, in Urdu Poetry, consists of a complex structure. Each sher/verse of a Ghazal can contain two different thoughts which make it difficult to classify. Two main factors that can be used for categorization of Ghazal text, as presented in [13-14], are content and the style. In Urdu poetry, each poet can have a varying degree of styles and themes. In this study, we have applied five different classification models and compared their results according to their accuracy scores. Also, we have analyzed the classifier performance by applying chi-2 and L1 based feature selection and observed changes in performance scores.

The rest of the paper is organized as follows. We discuss the background and related work in Section II, and present the methodology in Section III. Section IV presents the experimental setup, results, and discussions. Finally, the paper is concluded in Section V.

\section{BACKGROUND AND RELATED WORK}

In particular Urdu Poetry has many Principals such as Ghazal, Nazm, Hamd, Manaqbat etc but in this study we only focus on Ghazal but the same idea can be extended to other principals as well. Ghazal can consist of different, two liner couplets called Sher with same ending rhyme. Ghazals can have different themes which are mainly relevant to authors who have a different writing style for the same theme. There is a minimum of five couplets in each sher and couplets does not always have the same thoughts. Ghazals are one of the most difficult forms of poetry due to its writing parameters.

Research on automatic identification of Urdu poet is little to none. However, there are studies which use classification techniques to classify documents or text according to genres and authors for languages such English [13-15], Malay [3], Chinese [16], and Punjabi [17].

The Urdu language originated from Arabic language and various computational linguistic tasks like name entity recognition, part of speech tagging and machine translation have been performed on Urdu [18-22]. Fewer studies have been done to classify Arabic text of different categories such as sports, politics etc. using Decision Trees [23]. Work related to poetry classification has been done in different languages such as discussed in [23], which use SVM techniques to classify poetry according to themes and differentiate between poetry and nonpoetry text. Naïve Bayes [8-9], SVM [4-5], KNN [6], Decision Trees [7], and Random Forest [7] are more widely used supervised learning methods for classification. However, in text classification problem, SVM usually beats them all $[4,8,24]$.

\section{THE PROPOSED METHODOLOGY}

In order to perform the poet classification, we have adopted the methodology shown in Fig. 1. The Fig. 1 has three main sections namely Data Collection, Data

Mehran University Research Journal of Engineering \& Technology, Volume 38, No. 4, October, 2019 [p-ISSN: 0254-7821, e-ISSN: 2413-7219] 
Preprocessing and Poet Identification. Since no one has done a similar work in Urdu language, we need to prepare such data for the first time. For this, we have scraped Urdu ghazal data from various sites, tagged ghazals with their respective poets and stored in a database in the first section. In second section, we have applied few preprocessing techniques to convert it into an acceptable format. Afterward in Poet Identification section, we have applied some feature selection algorithms to choose only the important features. Finally, we have applied various machine learning classifiers to identify the poets.
Data Collection: In supervised machine learning algorithms, extensive training is required to develop a reasonable model. For this purpose, we wrote scrappers as well as collected data manually from different poetry websites, e.g. rakhta.pk, urdupoint.com. In total, we collected 3967 couplets of four different poets, having more than one million tokens with vocabulary size of 6427. Data were collected in CSF (Comma Separated File) and tagged manually. We collected Ghazals from the following poets: Mir Taqi Mir, Ahmed Faraz, Mirza Ghalib and Zafar Iqbal. These poets are the classes used to identify poets from our couplets data.

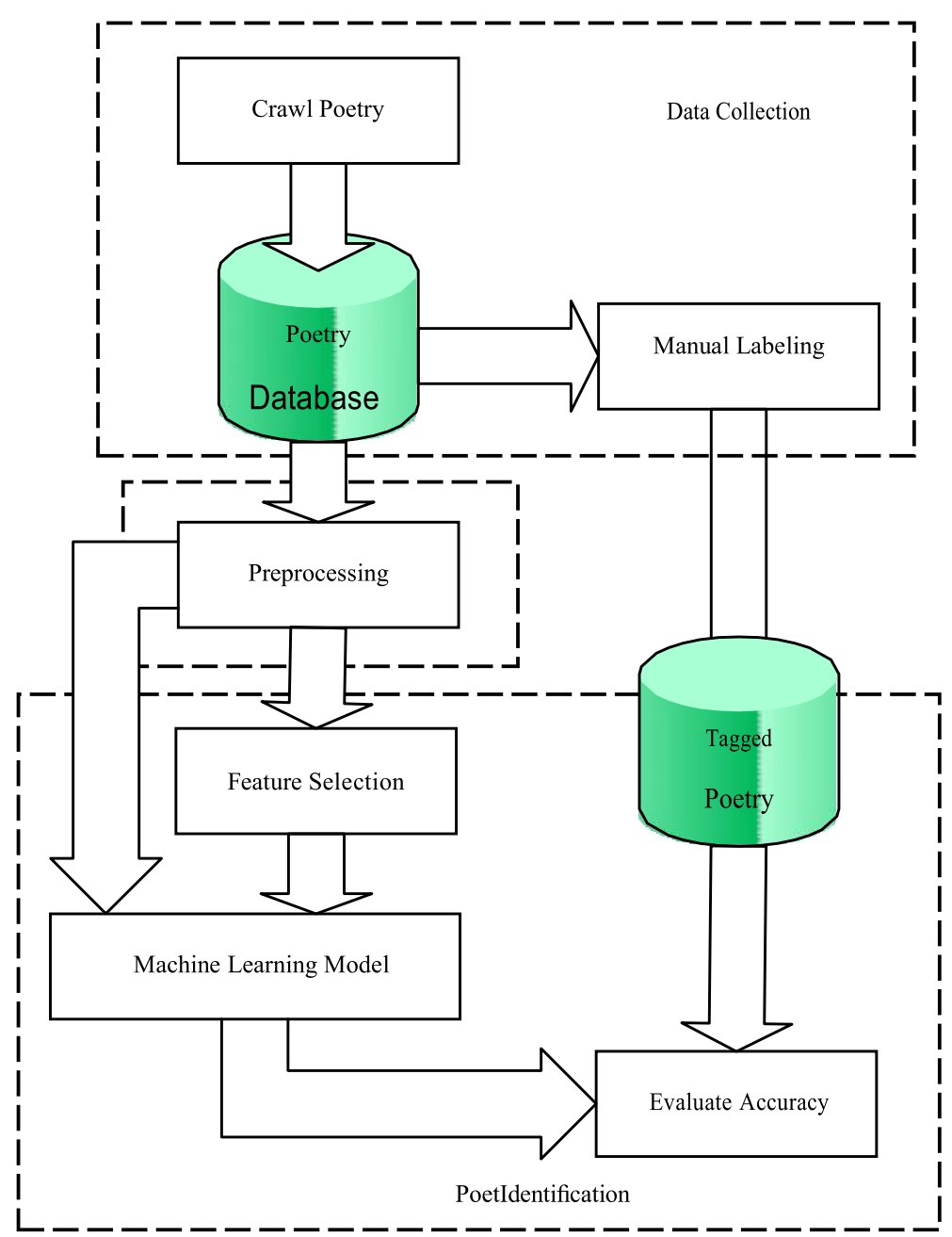

FIG. 1. THE PROPOSED ARCHITECTURE 
Preprocessing: We cannot directly apply any machine learning models on the collected data. We need to do some preprocessing to transform into an acceptable format. First, we apply tokenization to separate word from the couplets by using white space (space, tab, newline) as the delimiter. Then, we create the TDF (Term- DocumentFrequency) matrix, where each row in the matrix corresponds to a sher and each column corresponds to a term (word) in the sher. The preprocessing step can be explained by the following simple example. Let's say we have three sentences.

(1) He eats apple

(2) I am drinking water

(3) He eats food

As a first step, we build a unique vocabulary He, eats, apple, I, am, drinking, water, food from three sentences, thus the count is 8 unique words. In second step we build a TDF matrix of size $3 \times 8$, where 3 represents total number of documents and 8 represents unique words. In the last step, we will fill the matrix as shown in Table 1. Each entry of the Table 1 shows the number of occurrences of a word in the particular document.

Poet Identification: This section is further subdivided into sub-sections.

Feature Selection: After preprocessing step, we perform feature selection step to select the most relevant features for the poet identification. The feature selection methods applied are chi-square and L1-based. The reason to choose these methods is that the first is based on statistical and second is based on select from model approach. These both are the two widely used high level techniques for feature selection. The ChiSquare $\left(\chi^{2}\right)$ [25-27] is a feature selection method that computes the chi square statistics to evaluate the feature importance with respect to the classes. If the term and class are independent then its score is equal to 0 , otherwise 1 . A term with a higher chi-squared score is more informative. The L1-based feature selection method is based on linear regression where regression coefficients are penalized with L1 penalty forcing many of them to zero. The leftover non-zero coefficients are selected as features [28-29].

Applying Machine Learning Algorithms: Now, the data is in good shape to apply any machine learning algorithm. We have used five different machine learning classifiers for poet identification. The chosen classifiers cover a range of popular modes of classification: Naïve Bayes [89], decision trees [7], support vector machines [4-5,30], KNN [7] and Random Forest [7].

\section{EXPERIMENTS}

We have performed extensive experiments to evaluate our methodology and finalize the best classifier for such problem. The data were gathered both manually and

TABLE 1. TERM DOCUMENT FREQUENCYMATRIX

\begin{tabular}{|c|c|c|c|c|c|c|c|c|}
\hline Document & He & Eats & Apple & I & Am & Drinking & Water & Food \\
\hline 1 & 1 & 1 & 1 & 0 & 0 & 0 & 0 & 0 \\
\hline 2 & 0 & 0 & 0 & 1 & 1 & 1 & 1 & 0 \\
\hline 3 & 1 & 1 & 0 & 0 & 0 & 0 & 0 & 1 \\
\hline
\end{tabular}

Mehran University Research Journal of Engineering \& Technology, Volume 38, No. 4, October, 2019 [p-ISSN: 0254-7821, e-ISSN: 2413-7219] 
through scrappers. For evaluation, the data is divided into two parts: training data and testing data. Out of 3967 couplets, $80 \%$ of the documents are training dataset and $20 \%$ as a testing dataset as shown in Table 2 . We have performed three types of experiments i.e. poet identification without any feature selection; poet identification with Chi-2 feature selection; and poet identification with L1 based feature selection. The precision, recall and F1-measure scores in percentages are calculated to evaluate our five classifiers. Since we have a multi-class problem, F1-measure is considered as a more representative metric. Implementation of the system is done in Python language using scikit-learn [31] library.

Results: This section describes the results achieved after applying five different machine learning algorithms on the dataset with and without the two feature selection methods. The purpose of designing the experiments in this way is to evaluate the various set of features and machine learning models. Finally, we evaluate the best set of features along with the best model for poet identification.

The first set of experiments are performed along with the original set of features, that are extracted after applying the preprocessing and on the TDF matrix. The results for this set of experiments are summarized in Table 3.

The second set of experiments are performed after applying the Chi-2 feature selection algorithm to the TDF matrix. The Chi-2 feature selection algorithm selects the best features. The results for this set of experiments are summarized in Table 4.

The third set of experiments is performed after applying the L1 based feature selection algorithm to the TDF matrix. The results for this set of experiments are summarized in Table 5.

The overall F1-measure score for all the sets of experiments is further summarized in the plot shown in Fig. 2. The plot shows that SVM easily beats all the other classifiers, no matter feature selection algorithm is used or not. The second-best performing classifier is Naïve Bayes. The rest of the classifiers performed poorly. Even after applying the feature selection algorithms, the F1-measure score is not improved significantly in the case of Decision Tree, Random Forest and KNN classifier. The feature selection algorithm improved the F1-measure score reasonably for SVM and Naïve Bayes especially Naïve Bayes. Both the feature selection algorithms produce the overall best F1-measure, when SVM is used as a classifier.

TABLE 2. CORPUS DETAIL

\begin{tabular}{|c|c|c|c|}
\hline Poet & Total Sher & Training & Testing \\
\hline Mir Taqi Mir & 962 & 770 & 192 \\
\hline Ahmed Faraz & 959 & 766 & 207 \\
\hline Mirza Ghalib & 1033 & 826 & 203 \\
\hline Zafar Iqbal & 1013 & 810 & 795 \\
\hline Total & 3967 & 3172 & \\
\hline
\end{tabular}


Performance wise, KNN is the slowest and Naïve Bayes is the fastest classifier. The fast performance of the Naïve Bayes can be attributed to its simplistic computation [7]. SVM performs better and shows higher F1-measure score as it is flexible in term of errors and penalties and scale better for large data. SVM supports dense and sparse input in our context Urdu Poetry. Direct comparison with previous work is difficult due to limited work in Urdu linguistic and Urdu Poetry. However, since Urdu has its roots in Arabic language, therefore Urdu

TABLE 3. POET IDENTIFICATION (\%) WITHOUT FEATURE SELECTION

\begin{tabular}{|c|c|c|c|c|}
\hline Classification & Poets & Recall & Precision & F1-Measure \\
\hline \multirow{5}{*}{ SVM } & Faraz Ahmed & 64 & 66 & 64 \\
\hline & Mir Taqi Mir & 66 & 73 & 69 \\
\hline & Mirza Ghalib & 76 & 66 & 70 \\
\hline & Zafar Iqbal & 68 & 69 & 68 \\
\hline & Total & 69 & 69 & 69 \\
\hline \multirow{5}{*}{ Decision Tree } & Faraz Ahmed & 40 & 43 & 41 \\
\hline & Mir Taqi Mir & 48 & 48 & 48 \\
\hline & Mirza Ghalib & 50 & 39 & 43 \\
\hline & Zafar Iqbal & 39 & 46 & 42 \\
\hline & Total & 44 & 44 & 44 \\
\hline \multirow{5}{*}{ Random Forest } & Faraz Ahmed & 45 & 51 & 47 \\
\hline & Mir Taqi Mir & 47 & 64 & 54 \\
\hline & Mirza Ghalib & 58 & 39 & 46 \\
\hline & Zafar Iqbal & 54 & 46 & 49 \\
\hline & Total & 51 & 50 & 50 \\
\hline \multirow{5}{*}{ Naïve Bayes } & Faraz Ahmed & 45 & 67 & 53 \\
\hline & Mir Taqi Mir & 56 & 45 & 49 \\
\hline & Mirza Ghalib & 66 & 49 & 56 \\
\hline & Zafar Iqbal & 64 & 63 & 63 \\
\hline & Total & 58 & 56 & 57 \\
\hline \multirow{5}{*}{ K-Nearest Neighbor } & Faraz Ahmed & 36 & 48 & 41 \\
\hline & Mir Taqi Mir & 43 & 66 & 52 \\
\hline & Mirza Ghalib & 69 & 17 & 27 \\
\hline & Zafar Iqbal & 45 & 42 & 43 \\
\hline & Total & 48 & 43 & 45 \\
\hline
\end{tabular}


poetry classification results can be compared with previous research related to Arabic text classification.

Mesleh has applied chi-2 feature selection on Arabic text classification and then used SVM to get an average F-measure of $88.11 \%$ [26]. Similarly, Thabtah et. al. [32] have achieved an F-measure of $74 \%$ on Arabic text classification by using Naïve Bayes as machine learning algorithm and Chi-2 for feature selection. They both used Chi-2 for feature engineering and SVM and Naïve Bayes like us, and their F-measure is also similar to ours. These results show that our study align with the results obtained for automatic Arabic text classification.

TABLE 4. POET IDENTIFICATION (\%) WITH CHI -2 FEATURE SELECTION

\begin{tabular}{|c|c|c|c|c|}
\hline Classification & Poets & Recall & Precision & F1-Measure \\
\hline \multirow{5}{*}{ SVM } & Faraz Ahmed & 69 & 70 & 69 \\
\hline & Mir Taqi Mir & 72 & 75 & 73 \\
\hline & Mirza Ghalib & 79 & 71 & 74 \\
\hline & Zafar Iqbal & 70 & 73 & 71 \\
\hline & Total & 73 & 72 & 72 \\
\hline \multirow{5}{*}{ Decision Tree } & Faraz Ahmed & 42 & 49 & 45 \\
\hline & Mir Taqi Mir & 49 & 49 & 49 \\
\hline & Mirza Ghalib & 50 & 41 & 45 \\
\hline & Zafar Iqbal & 40 & 42 & 40 \\
\hline & Total & 45 & 45 & 45 \\
\hline \multirow{5}{*}{ Random Forest } & Faraz Ahmed & 45 & 52 & 48 \\
\hline & Mir Taqi Mir & 51 & 64 & 56 \\
\hline & Mirza Ghalib & 57 & 43 & 49 \\
\hline & Zafar Iqbal & 50 & 43 & 46 \\
\hline & Total & 51 & 50 & 50 \\
\hline \multirow{5}{*}{ Naïve Bayes } & Faraz Ahmed & 61 & 69 & 64 \\
\hline & Mir Taqi Mir & 57 & 50 & 53 \\
\hline & Mirza Ghalib & 71 & 48 & 57 \\
\hline & Zafar Iqbal & 62 & 82 & 70 \\
\hline & Total & 63 & 62 & 62 \\
\hline \multirow{5}{*}{ K-Nearest Neighbor } & Faraz Ahmed & 35 & 40 & 37 \\
\hline & Mir Taqi Mir & 43 & 71 & 53 \\
\hline & Mirza Ghalib & 62 & 32 & 42 \\
\hline & Zafar Iqbal & 53 & 38 & 44 \\
\hline & Total & 48 & 45 & 46 \\
\hline
\end{tabular}


Identification of Urdu Ghazal Poets using SVM

TABLE 5. POET IDENTIFICATION (\%) WITH L1 BASED FEATURE SELECTION

\begin{tabular}{|c|c|c|c|c|}
\hline Classification & Poets & Recall & Precision & F1-Measure \\
\hline \multirow{5}{*}{ SVM } & Faraz Ahmed & 69 & 69 & 69 \\
\hline & Mir Taqi Mir & 71 & 78 & 74 \\
\hline & Mirza Ghalib & 79 & 71 & 74 \\
\hline & Zafar Iqbal & 70 & 71 & 70 \\
\hline & Total & 72 & 72 & 72 \\
\hline \multirow{5}{*}{ Decision Tree } & Faraz Ahmed & 42 & 46 & 43 \\
\hline & Mir Taqi Mir & 42 & 46 & 43 \\
\hline & Mirza Ghalib & 55 & 46 & 50 \\
\hline & Zafar Iqbal & 43 & 44 & 43 \\
\hline & Total & 47 & 46 & 46 \\
\hline \multirow{5}{*}{ Random Forest } & Faraz Ahmed & 42 & 46 & 43 \\
\hline & Mir Taqi Mir & 48 & 58 & 52 \\
\hline & Mirza Ghalib & 60 & 43 & 50 \\
\hline & Zafar Iqbal & 51 & 51 & 51 \\
\hline & Total & 50 & 50 & 49 \\
\hline \multirow{5}{*}{ Naïve Bayes } & Faraz Ahmed & 77 & 61 & 68 \\
\hline & Mir Taqi Mir & 60 & 71 & 65 \\
\hline & Mirza Ghalib & 80 & 55 & 65 \\
\hline & Zafar Iqbal & 66 & 89 & 75 \\
\hline & Total & 71 & 69 & 70 \\
\hline \multirow{5}{*}{ K-Nearest Neighbor } & Faraz Ahmed & 41 & 35 & 37 \\
\hline & Mir Taqi Mir & 38 & 79 & 51 \\
\hline & Mirza Ghalib & 61 & 25 & 35 \\
\hline & Zafar Iqbal & 52 & 38 & 43 \\
\hline & Total & 48 & 44 & 46 \\
\hline
\end{tabular}

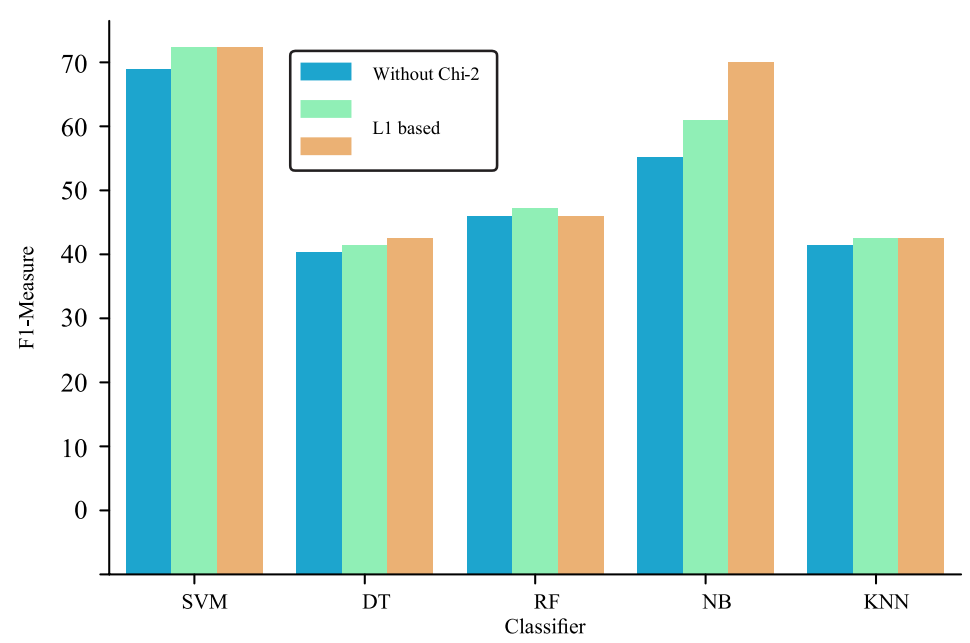

FIG. 2. GRAPHICAL REPRESENTATION OF CLASSIFIERS'PERFORMANCE 


\section{CONCLUSION}

Urdu poet identification is a challenging and interesting research. Unfortunately, it has been largely overlooked in the past studies. This study presents an experimental work on automatic identification of poets from Urdu poetry using five classification techniques i.e. SVM, Random Forest, KNN, Decision

Trees and Naïve Bayes. A collection of 4000 sher was gathered from various resources for experimenting with the aforementioned classification. Overall, the SVM performed the best and achieved 72\% F1-measure score. We have used two widely used feature selection techniques namely L1 based and chi-2 based feature selection algorithms. In the future, research will be carried out to identify suitable poetic features that may improve the reliability of the identification.

\section{ACKNOWLEDGMENT}

Authors are grateful to Prof. Dr. Syed Mansoor Sarwar, Principal, Punjab University College of Information Technology, Lahore, Pakistan, for providing infrastructure to perform extensive experiments.

\section{REFERENCES}

[1] Wikipedia Contributors, "Ghazal", Wikipedia, The Free Encyclopedia, 2018. [Online; accessed 23-September2018]. Qureshi, R.B., "Musical Gesture and Extra-Musical Meaning: Words and Music in the Urdu Ghazal", Journal of the American Musicological Society, Volume 43, No. 3, pp. 457-497, 1990.

[3] Jamal, N., Mohd. M., and Noah, S.A., "Poetry Classification Using Support Vector Machines", Journal of Computer Science, Volume 8, No. 9, pp. 1441-1446, 2012.
[4] Joachims, T., "Text Categorization with Support Vector Machines: Learning with Many Relevant Features", 10th European Conference on Machine Learning, Germany, April 21 - 24, 1998.

[5] Meyer, D., Leisch, F., and Hornik, K., "The Support Vector Machine under Test", Neuro-Computing, Volume 55, No. 1-2, pp. 169-186, 2003.

[6] Ghani, Y.Y.S., "A Study of Approaches to Hypertext Categorization", Journal of Intelligent Information Systems, Volume 18, pp. 219-241, 2002.

[7] Han, J., "Data Mining: Concepts and Techniques", Morgan Kaufmann Publishers Inc., San Francisco, CA, USA, 2005.

[8] Mccallum, A., and Nigam, K., "A Comparison of Event Models for Naive Bayes Text Classification", Learning for Text Categorization: AAAI Workshop, pp. 41-48, 1998.

[9] Rish, I., "An Empirical Study of the Naive Bayes Classifier", International Joint Conferences on Artificial Intelligence, Volume 3, pp. 41-46, New York, 2001.

[10] Shams, R., and Mercer, R.E., "Classifying SPAM Emails Using Text and Readability Features", IEEE 13th International Conference on Data Mining, pp. 657-666, December, 2013.

[11] Koppel, M., Schler, J., and Bonchek-Dokow, E., "Measuring Differentiability: Unmasking Pseudonymous Authors", Journal of Machine Learning Research, Volume 8, pp. 1261-1276, December, 2007.

[12] Usman, M., Shafique, Z., Ayub, S., and Malik, K., "Urdu Text Classification Using Majority Voting”, International Journal of Advanced Computer Science \& Applications, Volume 7, No. 8, pp. 265-273, 2016.

[13] Stamatatos, E., Kokkinakis, G., and Fakotakis, N., "Automatic Text Categorization in Terms of Genre and Author", Computational linguistics, Volume 26, No. 4, pp. 471-495, December, 2000.

[14] Finn, A., and Kushmerick, N., "Learning to Classify Documents According to Genre", Journal of the Association for Information Science and Technology, Volume 57, No. 11, pp. .1506-1518, 2006. 
[15] Kaplan, D.M., and Blei, D.M., “A Computational Approach to Style in American Poetry", $7^{\text {th }}$ IEEE International Conference on Data Mining, pp. 553-558, October, 2007.

[16] Voigt, R., and Jurafsky, D., "Tradition and Modernity in 20th Century Chinese Poetry”, 23rd International Conference on Computational Linguistics, 2013.

[17] Kaur, J., and Saini, J.R., "Automatic Punjabi Poetry Classification Using Machine Learning Algorithms with Reduced Feature Set”, International Journal of Artificial Intelligent Soft Computer, Volume 5, No. 4, pp. 311-319, January, 2017.

[18] Ali, A., Hussain, A., and Malik, M.K., "Model for EnglishUrdu Statistical Machine Translation", World Applied Sciences, Volume 24, pp. 1362-1367, 2013.

[19] Ali, W., Malik, M.K., Hussain, S., Siddiq, S., and Ali, A., "Urdu Noun Phrase Chunking: Hmm Based Approach", IEEE International Conference on Educational and Information Technology, Volume 2, pp. V2-494, 2010.

[20] Karamat, N., Malik, K., and Hussain, S., "Improving Generation in Machine Translation by Separating Syntactic and Morphological Processes", IEEE Conference on Frontiers of Information Technology, pp. 195-200, 2011.

[21] Malik, M.K., and Sarwar, S.M., "Urdu Named Entity Recognition System Using Hidden Markov Model", Pakistan Journal of Engineering and Applied Sciences, 2017.

[22] Mateen, A., Malik, M.K., Nawaz, Z., Danish, H., Siddiqui, M.H., and Abbas, Q., "A Hybrid Stemmer of Punjabi Shahmukhi Script", International Journal of Computer Science \& Network Security, Volume 17, No. 8, pp. 90-97, 2017.

[23] A1-Diabat, F., “Arabic Text Categorization Using Classification Rule Mining", Applied Mathematical Sciences, Volume 6, No. 81, pp. 14033-4046, 2012
[24] Wiss, S.M., Aptc, C., Damerau, F.J., Johnson, D.E., Oles, F.J., Goetz, T., and Hampp, T., "Maximizing TextMining Performance", IEEE Intelligent Systems, Volume 14, No. 4, pp. 63-69, July, 1999.

[25] Hall, M.A., and Smith, L.A., "Practical Feature Subset Selection for Machine Learning", Australasian Computer Science Conference, 998.

[26] Mesleh, A.M., "Chi Square Feature Extraction Based SVMS Arabic Language Text Categorization System", Journal of Computer Science, Volume 3, No. 6, pp. $430-435,2007$.

[27] Thabtah, F., Eljinini, M.A.H., Zamzeer, M., and W.M., "Naïve Bayesian Based on Chi Square to Categorize Arabic Data", Communications of the IBIMA, Volume 10, 2009.

[28] Bach, F.R., "Bolasso: Model Consistent Lasso Estimation through the Bootstrap", Proceedings of 25th ACM International Conference on Machine Learning, pp. 33-40, 2008

[29] Zare H. Haffari, G., Gupta, A., and Brinkman, PO.R., "Scoring Relevancy of Features Based on Combinatorial Analysis of Lasso with Application to Lymphoma Diagnosis", BMC Genomics, Volume14, pp. S1-S14, 2013.

[30] Odeh, A., Abu-Errub, A.M., Shambour, Q., and Turab, N., "Arabic Text Categorization Algorithm Using Vector Evaluation Method", Computing Research Repository, 2015.

[31] Butinick, I., Louppe, G., Blondel, M., Pedregosa, F., Mueller, A., Grisel, O., Niculaf, F., Prettenhofer, P., Gramfort, A., Grobler, J. Layton, R., Vanderplas, J., Joly, A., Holt, B., and Varoquaux, G., "API Design for Machine Learning Software: Experiences from the Scikit-Learn Project”, European Conference on Machine Learning and Principles and Practice of Knowledge Discovery in Databases, pp. 108-122, 2013.

[32] Thabtah, F., Eljinini, M., Zamzeer, M., and Hadi, W., "Naïve Bayesian Based on Chi Square to Categorize Arabic Data", Proceedings of $11^{\text {th }}$ International Conference on Innovation and Knowledge Management in Twin Track Economies, pp. 4-6, Cairo, Egypt, 2009 